\title{
Through-grid wicking enables high-speed cryoEM specimen preparation
}

Yong Zi Tan ${ }^{1}$ and John Rubinstein ${ }^{2}$

${ }^{1}$ The Hospital for Sick Children, United States, ${ }^{2}$ The Hospital for Sick Children, Canada

Blotting times for conventional cryoEM specimen preparation complicate time-resolved studies and lead to some specimens adopting preferred orientations or denaturing at the air-water interface ${ }^{1}$. Here, it is shown that solution sprayed onto one side of a holey cryoEM grid can be wicked through the grid by a glass-fiber filter held against the opposite side, often called the 'back', of the grid, producing a film suitable for vitrification (Figure 1). This process can be completed in tens of milliseconds. Ultrasonic specimen application and through-grid wicking were combined in a high-speed specimenpreparation device that was named 'Back-it-up' or BIU ${ }^{2}$. Similar to the Shake-it-off platform on which it is based ${ }^{3}$, the BIU instrument is inexpensive to construct. The implementation described here cost $\sim \mathrm{C} \$ 1,000$ ( USD\$700) to build. All components needed to build the device are either commercially available or can be made by online 3D printing, printed circuit board fabrication, and CNC milling services, so that specialized manufacturing infrastructure is not required. The high liquid-absorption capacity of the glass fiber compared with self-wicking grids makes the method relatively insensitive to the amount of sample applied. Consequently, through-grid wicking produces large areas of ice that are suitable for cryoEM for both soluble (apoferritin was determined to $2.0 \AA$ resolution, Figure 1) and detergent-solubilized protein complexes (Saccharomyces cerevisiae 4 and Mycobacterium smegmatis $^{5}$ ATP synthases). The speed of the device increases the number of views for a specimen that suffers from preferred orientations. This was shown using influenza A hemagglutinin trimer, a protein known to undergo time-dependent adoption of a preferred top-view orientation at the air-water interface during grid freezing ${ }^{6}$ (Figure 2). Side views of the particles was increased from $2 \%$ (conventional CP3 blotting) to $34 \%$ using BIU, and enabled the reconstruction of a directionally isotropic map at $2.9 \AA$ resolution with a 3DFSC sphericity of $0.92^{7}$. We believe that the reproducibility and large areas of usable ice from through-grid wicking, combined with the simplicity and low construction cost of the BIU device, provides a robust and easily accessible approach to high-speed cryoEM grid preparation for a variety of specimens.

\section{References}

${ }^{1}$ Glaeser, R.M., 2021. Preparing Better Samples for Cryo-Electron Microscopy: Biochemical Challenges Do not End with Isolation and Purification. Annual Review of Biochemistry, 90.

${ }^{2}$ Tan, Y.Z. and Rubinstein, J.L., 2020. Through-grid wicking enables high-speed cryoEM specimen preparation. Acta Crystallographica Section D: Structural Biology, 76(11).

${ }^{3}$ Rubinstein, J.L., Guo, H., Ripstein, Z.A., Haydaroglu, A., Au, A., Yip, C.M., Di Trani, J.M., Benlekbir, S. and Kwok, T., 2019. Shake-it-off: a simple ultrasonic cryo-EM specimen-preparation device. Acta Crystallographica Section D: Structural Biology, 75(12), pp.1063-1070.

${ }^{4}$ Guo, H., Suzuki, T. and Rubinstein, J.L., 2019. Structure of a bacterial ATP synthase. Elife, 8, p.e43128. ${ }^{5}$ Guo, H., Courbon, G.M., Bueler, S.A., Mai, J., Liu, J. and Rubinstein, J.L., 2021. Structure of mycobacterial ATP synthase bound to the tuberculosis drug bedaquiline. Nature, 589(7840), pp.143-147. 


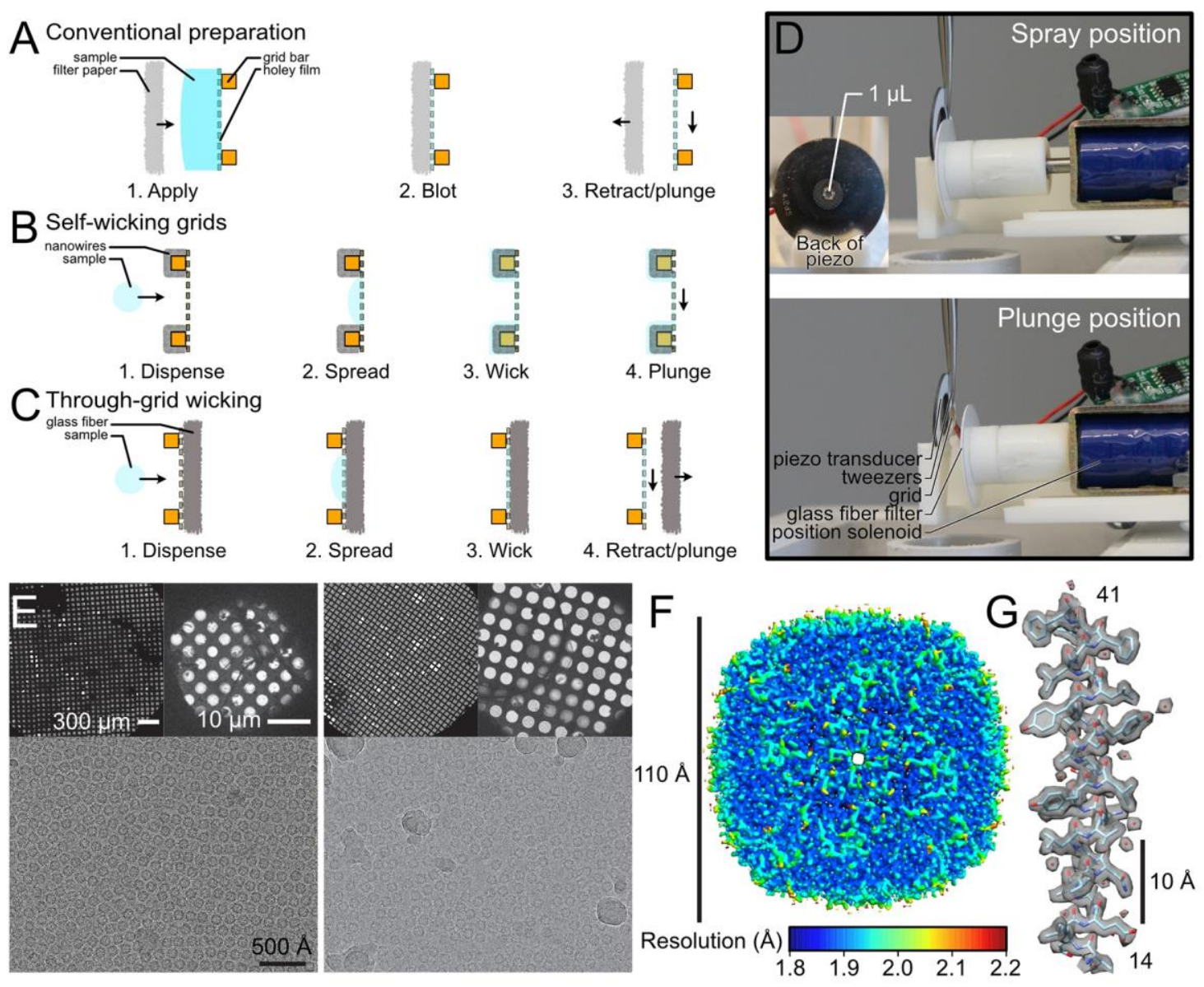

Figure 1. Through-grid wicking enables high-resolution structure determination. (A) Conventional specimen preparation: sample is applied to the grid and blotted with filter paper to produce a thin film suitable for plunge freezing. (B) Self-wicking grids: nanowires on the grid bars absorb excess liquid from small droplets to produce a thin film. (C) Through-grid wicking: sample is applied to one side of the grid while a glass fiber filter on the other side removes excess liquid to produce a thin film. (D) Experimental implementation of through-grid wicking in the BIU device. (E) Grid atlas, grid square, and high magnification images of human apoferritin prepared with BIU. (F) Local resolution for the apoferritin reconstruction. (G) A representative $\alpha$ helix from the apoferritin model (residues 14 to 41) and experimental density map (grey surface). Water molecules are displayed as red spheres.

${ }^{6}$ Noble, A.J., Wei, H., Dandey, V.P., Zhang, Z., Tan, Y.Z., Potter, C.S. and Carragher, B., 2018. Reducing effects of particle adsorption to the air-water interface in cryo-EM. Nature methods, 15(10), pp.793-795. ${ }^{7}$ Tan, Y.Z., Baldwin, P.R., Davis, J.H., Williamson, J.R., Potter, C.S., Carragher, B. and Lyumkis, D., 2017. Addressing preferred specimen orientation in single-particle cryo-EM through tilting. Nature methods, 14(8), pp.793-796. 




Figure 2. High-speed freezing with BIU improves the orientation distribution in a hemagglutinin trimer specimen. (A) Timing of the grid freezing process measured with high-speed video. (B) The complete set of 2D class averages from hemagglutinin trimer specimens prepared with the Gatan CP3 and BIU. The fraction of particle images contributing to top and oblique view classes versus side view classes is indicated. (C) Local resolution map of the hemagglutinin trimer. (D) A representative $\alpha$ helix (residues 405-454), $\beta$ sheet (residue 154-175, 195-228, 238-251), and N-glycan (residue 165) from the hemagglutinin model, overlaid with the experimental density map (grey surface). 